\title{
Saturation Effects In Degenerate Phase Sensitive Fiber Optic Parametric Amplifiers
}

\author{
Joseph Kakande ${ }^{(1)}$, Francesca Parmigiani ${ }^{(1)}$, Radan Slavík ${ }^{(1)}$, Lars Grüner-Nielsen ${ }^{(2)}$, Dan Jakobsen ${ }^{(2)}$, \\ Søren Herstrøm ${ }^{(2)}$, Periklis Petropoulos ${ }^{(1)}$ and David J. Richardson ${ }^{(1)}$ \\ (1) Optoelectronics Research Centre, University of Southampton, Southampton, SO17 1BJ, UK \\ Tel.:+44-2380594524, Fax +44-2380593142, e-mail:jkk@orc.soton.ac.uk \\ (2) OFS, Priorparken 680, 2605 Brøndby, Denmark.
}

\begin{abstract}
We experimentally study saturation effects in degenerate phase sensitive amplifiers, revealing and explaining a gain regime suitable for all-optical signal processing functions such as phase regeneration.
\end{abstract}

\section{Introduction}

Four wave mixing (FWM) based phaseinsensitive fiber optical parametric amplifiers (PIFOPAs) when operated in the linear gain regime have been demonstrated to achieve low (albeit quantum limited) noise ${ }^{1}$, flat, high and wide gain bandwidths ${ }^{2}$ exceeding those achievable using the ubiquitous erbium doped fibre amplifier (EDFA). To date though, these devices have been plagued by detrimental effects such as stimulated Brillouin scattering (SBS), cross gain modulation (XGM) via pump depletion and intrachannel crosstalk due to spurious four wave mixing products. PI-FOPAs can also be operated in the nonlinear gain regime to perform all-optical amplitude processing of input signals. The predominant effect utilised is gain saturation which can be used to perform amplitude regeneration of high speed signals ${ }^{3-5}$.

The recent resurgence of interest in the use of the entire complex field for optical signalling has meant that the capability of the FOPA to manipulate signal phase is becoming increasingly relevant. Phase sensitive (PS) operation can be achieved by having the signal and idler fields present at the input to the FOPA. In particular, in the degenerate PS- FOPA, two strong pumps spaced equally apart in frequency from a signal wave (in this case the signal and idler fields can be considered to be at the same frequency). Degenerate PS amplification is characterised by a sinusoidal phase-toamplitude transfer function and a step phase-tophase transfer characteristic ${ }^{6}$. This configuration is particularly useful because the gain (and phase step) is periodic in $180^{\circ}$ with the signal phase, which is ideal for phase regeneration of binary phase shift keyed (BPSK) optical signals ${ }^{7,8}$. Achieving saturation via increasing pump and signal powers in FOPAs is often limited by the SBS thresholds of the highly nonlinear fibre (HNLF) used. For PI-FOPAs, the use of phase dithering to increase the SBS threshold is the most common solution to this problem. In PS-FOPAs however, phase dithering is not desirable as it distorts the signal phase, limiting the corresponding regenerative properties. Short lengths of highly nonlinear Bismuth-Oxide fibre have previously been used to experimentally study the gain properties of the degenerate PS-FOPA ${ }^{7}$. In this paper, we utilise a novel high SBS threshold HNLF to experimentally study the nature and cause of saturation effects in the degenerate PS-FOPA configuration.

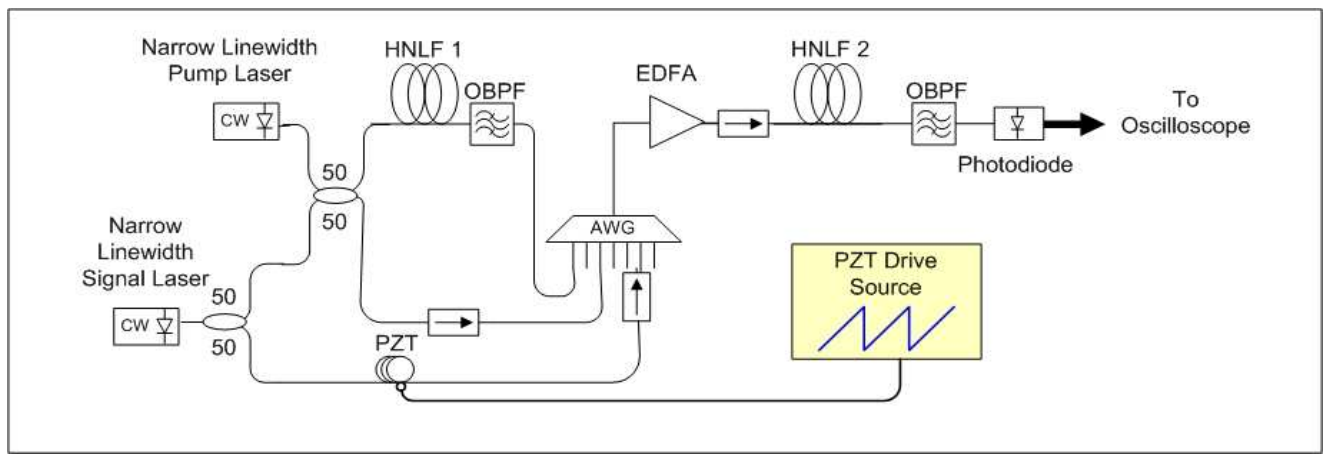

Fig. 1: Experimental setup of degenerate PS-FOPA. OBPF: Optical Bandpass Filter 


\section{Experimental setup and results}

The experimental setup is shown in Fig. 1. Two narrow linewidth signals $200 \mathrm{GHz}$ apart were mixed in a Ge-doped HNLF (OFS, Denmark) generating an idler wave. The HNLF length, dispersion, nonlinear coefficient and attenuation were $500 \mathrm{~m}, 0.09 \mathrm{ps} / \mathrm{nm} / \mathrm{km}, 11.5 \mathrm{~W}^{-1} \mathrm{~km}^{-1}$ and

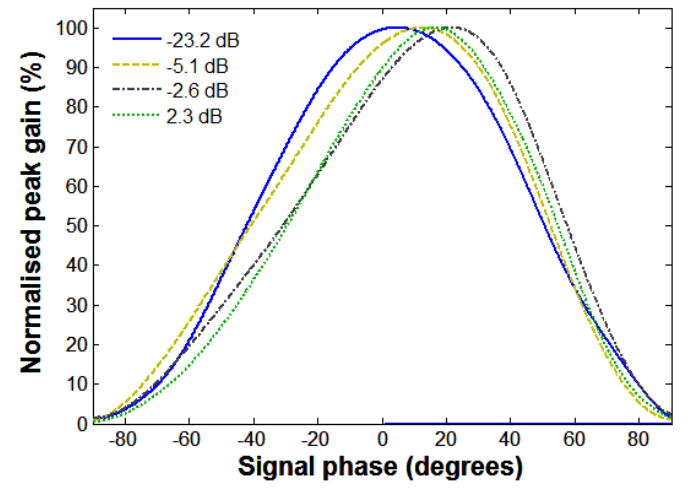

Fig. 2: Saturation characteristics as signal-to-pumps ratio is varied for PS-FOPA (total power of $29 \mathrm{dBm}$ ).

$0.83 \mathrm{~dB} / \mathrm{km}$ respectively. The idler was filtered out, in so doing generating a third wave locked in frequency and phase to the two signals at the HNLF input. The three waves spaced $200 \mathrm{GHz}$ apart could then be used as the two pumps and signal for the PS-FOPA. A variable attenuator allowed control of the signal power relative to the pumps. The degenerate PS-FOPA utilised a novel high SBS threshold strained aluminosilicate HNLF (OFS, Denmark). The length, dispersion, polarization mode dispersion, nonlinear coefficient and attenuation of the fiber were $177 \mathrm{~m},-0.13 \mathrm{ps} / \mathrm{nm} / \mathrm{km}, 0.11 \mathrm{pskm}^{-0.5}, 7.1 \mathrm{~W}^{-}$ ${ }^{1} \mathrm{~km}^{-1}$ and $15 \mathrm{~dB} / \mathrm{km}$, respectively. The high SBS threshold meant that in excess of $1 \mathrm{~W}$ per pump could be coupled into the fibre.

The phase of the signal wave was modulated using a $50 \mathrm{kHz}$ ramp function applied to a piezoelectric fibre stretcher. The choice of the ramp frequency was chosen to correspond to the resonant frequency of the piezo-electric stretcher used, allowing the phase to be swept by many multiples of $180^{\circ}$ for a peak-to-peak drive voltage of under $100 \mathrm{~V}$. The three waves were then combined, amplified in a high power polarization maintaining EDFA to a fixed total power, and then launched into the degenerate PS-FOPA. Following the parametric interaction, the signal at the PS-FOPA output was filtered, attenuated and coupled into a low power photodiode. The electrical signal from the photodiode was then observed using a high speed digital oscilloscope. Post-processing was then carried out to eliminate high-frequency noise and normalise the gain curves.

The normalised signal gain curves for a total power of $29 \mathrm{dBm}$ (pumps and signal combined) coupled into the fibre are shown in Fig. 2. At this moderate pump power, for low input signal

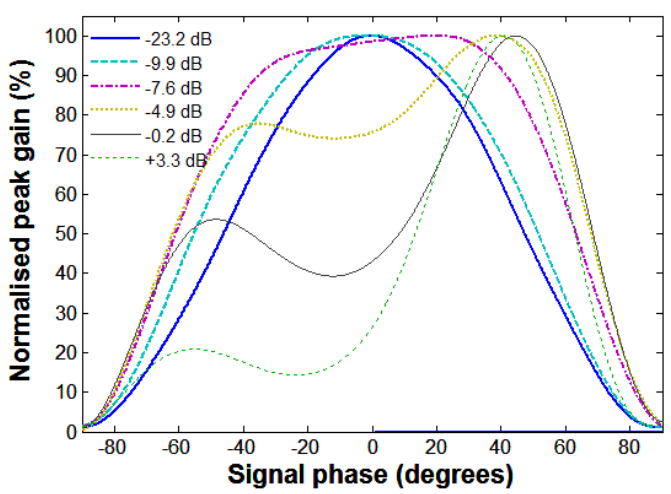

Fig. 3: Saturation characteristics as signal-to-pumps ratio is varied for PS-FOPA (total power of $32 \mathrm{dBm}$ ).

levels (solid curve in Fig. 2) the gain curve is sinusoidal with relative signal input phase as expected from theory 9 . As the signal power increases, the curves accumulate a skew, with the gain peak seemingly detuned in phase from the central phase position. Because each curve is captured for a fixed signal power at the PSFOPA input, this differs from $\mathrm{PI}$ saturation because a nonlinear dependence on input signal phase exists. In this case, every point on the curve experiences a gain shift due to the alteration of the phase matching condition by the variation in the signal/pump power proportions during propagation. As the signal and pumps propagate down the fibre in which the PS interaction occurs, at every point a nonlinear contribution to the phase matching exists as a result of self- and cross-phase modulation (SPM and XPM respectively). Because the gain is dependent on the signal phase, the absolute powers and therefore the nonlinear phase matching varies with the input phase. For strong signal levels, this phase dependent behaviour leads to a modification of the gain characteristic, and ultimately to gain saturation.

For applications such as the regeneration of binary phase shift keyed (BPSK) signals, a crucial requirement would be that the PS gain curves have a flat-top, minimising the transfer of phase to amplitude noise. To obtain such flattening, it was necessary to increase the total power coupled into the PS-FOPA to $32 \mathrm{dBm}$. A selection of the resulting gain curves are shown in Fig. 3. At the lowest signal level assessed a sinusoidal gain curve is obtained indicating the absence of significant gain saturation (thick solid line in Fig. 3). As the signal power is increased, the gain curve is seen to acquire a flat-top (dashed-dotted line in Fig. 3). Increasing the signal power further reveals that the flattening is 
in fact due to the growth of a second peak in the gain curve that detunes in phase from the central position as the signal level is increased.

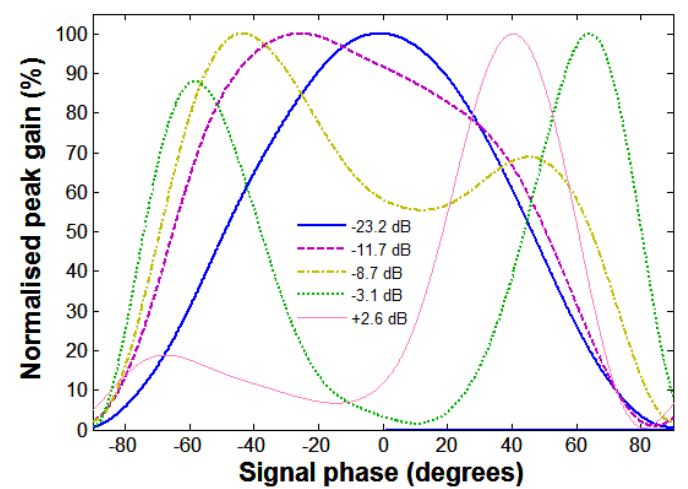

Fig. 4: Saturation characteristics as signal-to-pumps ratio is varied for PS-FOPA (total power of $33 \mathrm{dBm}$ ).

The total signal power was then increased further to $33 \mathrm{dBm}$ and the gain curves obtained are shown in Fig. 4. The gain curve goes from sinusoidal at low signal levels to skewed at moderate power levels, as previously discussed. At high signal levels, though, it is possible to have two separate peaks in the gain curve for a $180^{\circ}$ change in the input signal phase, contrasting with a single peak for lower power levels. This can be seen in the dotted line in Fig. 4. This points further towards the saturation effect stemming from a modification of the nonlinear phase matching condition and not just from pump power depletion. For $0^{\circ}$ input phase as an example, the gain goes from maximum for the signal $23.2 \mathrm{~dB}$ lower than the pumps, to minimum at $-3.1 \mathrm{~dB}$, and then starts to increase again for stronger signal levels. The ability to tune the periodicity of the phase response as well as the peak gains of a PSA by saturation opens up a whole new host of applications, an example being the phase regeneration of QPSK and M-PSK signals.

In the dotted line in Fig. 4, the peaks have a $14 \%$ difference in power. To obtain two equal peaks, the signal level and the total power into the PSA were varied and the results are shown in Fig. 5. Generating dual peaks can be achieved by increasing the signal level beyond $8 \mathrm{~dB}$ in proportion to the total power coupled into the PSA. It is also worth pointing out that for a total power of $32 \mathrm{dBm}$ (Fig. 3 thin solid line) the spacing between the peaks was approximately $90^{\circ}$, despite a peak to peak variation of $50 \%$. At $33 \mathrm{dBm}$ (dotted line Fig. 5) the spacing between the peaks was $70^{\circ}$ without significantly compromising the phase sensitive swing (97\%). Finally moving to $34 \mathrm{dBm}$ (solid line Fig. 5) this reduced to $52^{\circ}$ with equal gain peaks. These studies suggest that by optimising the signal and pump powers further it should be possible to achieve the desired gain shape, such as the $90^{\circ}$ spacing required for regeneration of QPSK signals in a single PS-FOPA.

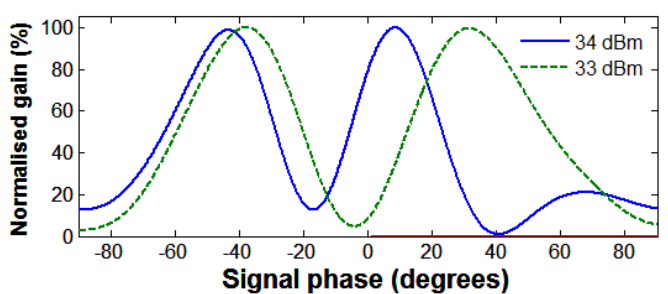

Fig. 5: PS-FOPA saturation optimised to achieve two gain peaks within a $180^{\circ}$ signal phase period.

\section{Conclusions}

We have experimentally studied saturation effects in PS-FOPAs showing the impact of the nonlinear phase matching term on the gain characteristics. The ability to experimentally access this high saturation regime stems from the very high SBS threshold levels for the HNLF used, allowing access to a flat top regime suitable for BPSK phase regeneration, as well as a further saturation regime characterised by multiple gain peaks within a $180^{\circ}$ signal phase periodicity.

\section{Acknowledgements}

We would like to thank OFS Denmark and Eblana Photonics Inc., Dublin, Ireland, for respectively providing HNLFs and lasers used in the experiment. This research has received funding from the European Communities Seventh Framework Programme FP/2007-2013 under grant agreement 224547 (PHASORS).

\section{References}

[1] A. Durecu-Legrand et al., Photonics Technology Letters, IEEE 17, 1178 (2005).

[2] J. M. C. Boggio et al., Photonics Technology Letters, IEEE 21, 612 (2009).

[3] C. J. McKinstrie, S. Radic, and A. H. Gnauck, Opt. Photon. News 18, 34 (2007).

[4] K. Inoue, Electronics Letters 36, 1016 (2000). [5] P. Kylemark et al., Lightwave Technology, Journal of 24, 3471 (2006).

[6] A. Bogris, and D. Syvridis, leee Photonics Technology Letters 18, 2144 (2006).

[7] K. Croussore, and G. F. Li, IEEE Photonics Technology Letters 19, 864 (2007).

[8] Parmigiani F. et al. Proc. OFC'10, PDPC3 (2010).

[9] R. Tang et al., Optics Express 13, 10483 (2005). 